\title{
An Efficient Method of Multi-robots Topological Map Building \\ Wang $\mathrm{Na}$, Wang Haiyan, Liu Jixin, Zheng Yi \\ Qingdao Huanghai College Qingdao China \\ 187262174@qq.com, 541101645@qq.com, 25669057@qq.com, 121663260@qq.com
}

\begin{abstract}
In order to accomplish task, map building is the key technology in mobile robotic control in unknown environment. In this paper, a mathematical model of map integration is established to solve multi-robot topological map integration problem. A new method which was a combination of the classical ICP (Iterative Closest Point) algorithm and the singular value decomposition (SVD) algorithm was used. The simulation results showed that the ICP-SVD image registration algorithm applied to the topology map fusion made the algorithm implementation become more simple. What more, the model also reduced time consuming, and the matching results were better.
\end{abstract}

Keywords: Multi-robots; Topological Map; Image registration; ICP-SVD

\section{Introduction}

Map building is the key to the robot control and navigation in unknown environment. The fusion of multi-robots' sensor data can effectively improve the accuracy of the environment map. Topological map is the combination of environment's nodes and connecting lines. So, topological map reduces information storage memory and this will improve computational efficiency.

\section{Raising a question}

In recent research, Dedeoglu solved the problem of the road map fusion, using a vertex matching between two maps. Then he estimated a transformation scheme, matched the other vertices, and generated a unified global map. However, multi-robot cooperation has not got a complete solution. Konolige used a method of decision-making framework theory, so he realized robots' relative positioning within the local map. This proved that the fusion method based on feature points has higher efficiency. But the disadvantage is that the extraction process of feature points is more complicated.

This paper uses ICP (Iterative Closest Point) image registration method, and this method was first proposed by Besl in 1992. In this paper, topological map fusion comes from geometric information, such as connectivity path, node type, etc. According to image registration of geometric feature, it retains the best matching scheme based on the size of matching range and error. So finally it merged the two maps.

\section{The establishment of mathematical model}

First, convert the topological map, it uses the rigid body transformation of linear transformation.

Show the point with homogeneous coordinates. Vector $[\mathrm{x}, \mathrm{y}, 1]^{\mathrm{T}}$ express point $\mathrm{p}(\mathrm{x}, \mathrm{y})$, $\mathrm{R}=\left[\begin{array}{cc}\cos \theta & -\sin \theta \\ \sin \theta & \cos \theta\end{array}\right]$ express rotation matrix, $\mathrm{T}=\left[\begin{array}{l}\mathrm{t}_{\mathrm{x}} \\ \mathrm{t}_{\mathrm{y}}\end{array}\right]$ express translational matrix, and $\mathrm{t}_{\mathrm{x}}, \mathrm{t}_{\mathrm{y}}, \theta$ respectively express the $\mathrm{x}$ direction, $\mathrm{y}$ direction translation and counterclockwise rotation angle.

Make ${ }^{t_{x}, t_{y}, \theta}$ be three real Numbers. So point $p$ transfers to as follows relating to ${ }^{t_{x}, t_{y}, \theta}$ :

$$
\mathrm{TR}_{\mathrm{t}_{\mathrm{x}}, \mathrm{t}, \mathrm{\theta}, \theta}(\mathrm{x}, \mathrm{y})=\left[\begin{array}{lll}
1 & 0 & 0 \\
0 & 1 & 0
\end{array}\right]\left[\begin{array}{ccc}
\cos \theta & -\sin \theta & \mathrm{t}_{\mathrm{x}} \\
\sin \theta & \cos \theta & \mathrm{t}_{\mathrm{y}} \\
0 & 0 & 1
\end{array}\right]\left[\begin{array}{l}
\mathrm{x} \\
\mathrm{y} \\
1
\end{array}\right]
$$

Formula (1) shows $\theta$ rotation transform of point $\mathrm{p}(\mathrm{x}, \mathrm{y}),\left(\mathrm{t}_{\mathrm{x}}, \mathrm{t}_{\mathrm{y}}\right)$ parallel motion along the axis direction.

Topological map merging uses the method of two matches. If two local maps are known, the 
purpose is to search a plane transformation, when the corresponding matching scope is the biggest.

The two maps are written as MapA and MapB. EA、EB express two matrices of these maps. The elements in the matrix express path endpoint coordinates. Assuming that path number is $\mathrm{n}$ and $\mathrm{m}$ respectively. The similarity between MapA and MapB as follows:

$$
\begin{gathered}
\omega\left(\operatorname{Map}_{A}, M a p_{B}\right)=\sum_{i=1}^{n} \sum_{j=1}^{m} E q\left(E_{A}[i], E_{B}[j]\right) \\
E q(a, b)=\left\{\begin{array}{cc}
1 & a=b \\
0 & \text { otherwise }
\end{array}\right.
\end{gathered}
$$

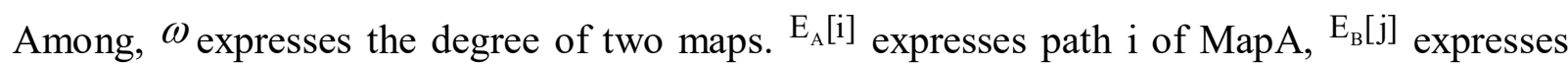
path $\mathrm{j}$ of MapB.

\section{Steps of ICP-SVD algorithm}

ICP - SVD algorithm applied to the topological map merging, the basic input is the set of path endpoints of two maps. Then it searches the nearest point on the fixed map for each path endpoint.

The set of path endpoints of the fixed map is called $\mathrm{M}$, and the coordinates are as follows: $\left\{M_{i} \mid M_{i} \in R^{2}, i=1,2, \ldots, N_{m}\right\}$.

Another set of path endpoints of the map for plane transformation is called $\mathrm{D}$, and the coordinates are as follows: $\left\{\mathrm{D}_{\mathrm{i}} \mid \mathrm{D}_{\mathrm{i}} \in \mathrm{R}^{2}, \mathrm{i}=1,2, \ldots, \mathrm{N}_{\mathrm{d}}\right\}$.

When the kth iteration is calculated, $\left\{D_{i}{ }^{k} \mid D_{i}{ }^{k} \in R^{2}, i=1,2, \ldots, N_{d}\right\}$, it computes the transformation matrix between $M$ and $D^{k}$. Then it will update the original transform to minimize the distance between the two sets of data. That means the following optimization function is minimized by iteration:

$$
f(\mathrm{R}, \mathrm{T})=\sum_{\mathrm{i}=1}^{\mathrm{N}_{\mathrm{m}}} \sum_{\mathrm{j}=1}^{\mathrm{N}_{\mathrm{d}}}\left\|\mathrm{M}_{\mathrm{i}}-\left(\mathrm{R} \times \mathrm{D}_{\mathrm{j}}+\mathrm{T}\right)\right\|^{2}
$$

Each iteration of ICP-SVD algorithm includes the following four steps:

Step1: Initialization. Calculate the mean of the two sets of points: $U_{m}=\frac{1}{N_{m}} \sum_{i=1}^{N_{m}} M_{i}, U_{d}=\frac{1}{N_{d}} \sum_{j=1}^{N_{d}} D_{j}$. Initialize the rotation matrix $R=\left[\begin{array}{ll}1 & 0 \\ 0 & 1\end{array}\right]$, translation matrix $\mathrm{T}=\left[\mathrm{U}_{\mathrm{m}}-\mathrm{U}_{\mathrm{d}}\right]$, so $\mathrm{D}^{\prime}=\mathrm{R} \times \mathrm{D}+\mathrm{T}$;

Step2: The rotation matrix and translation matrix are calculated by using the SVD algorithm.

Suppose that $d$ is the center of point set $D, m$ is the center of point set M. Subtract the barycentric coordinates from all the points. Then the points after translation are: $M_{i}^{\prime}=M_{i}-m$, $\mathrm{D}_{\mathrm{i}}{ }^{\prime}=\mathrm{D}_{\mathrm{i}}-\mathrm{d}$. The total error after translation is:

$$
\mathrm{E}=\sum_{\mathrm{i}=1}^{\mathrm{n}}\left\|\mathrm{M}_{\mathrm{i}}^{\prime}-\mathrm{RD}_{\mathrm{i}}^{\prime}\right\|^{2}
$$

Minimize $\mathrm{E}$ is equivalent to

Calculate $\mathrm{H}$ using $\mathrm{SVD}, \mathrm{H}=\mathrm{U} \Lambda \mathrm{V}, \mathrm{X}=\mathrm{UV}^{\mathrm{T}}$, if $\operatorname{det}(\mathrm{X})=1$, so $\mathrm{U}$ and $\mathrm{V}$ are two orthogonal $\sum_{i=1}^{n} M_{i}{ }^{T} R D_{i}^{\prime}=\operatorname{Trace}\left(R \sum_{i=1}^{n} M_{i}^{\prime} D_{i}^{\prime T}\right)=\operatorname{Trace}(R H)$

Among them, $\mathrm{H}=\sum_{\mathrm{i}=1}^{\mathrm{n}} \mathrm{M}_{\mathrm{i}}{ }^{\prime} \mathrm{D}_{\mathrm{i}}^{\mathrm{T}}$. matrices. Then get that, $\mathrm{R}=\mathrm{X}$, translation vector $\mathrm{T}=\mathrm{m}-\mathrm{R} \times \mathrm{d}$;

Step3: Apply $\mathrm{R}$ and $\mathrm{T}$ to the point set $\mathrm{D}$, then obtain the new point set $\mathrm{D}^{\prime}=\mathrm{R} \times \mathrm{D}+\mathrm{T}$, and instead of the original D;

Step4: Calculate the target function $f(\mathrm{R}, \mathrm{T})$ between new point set $\mathrm{D}$ and $\mathrm{M}$. If two iteration errors are less than the given threshold $\tau$ (In this paper $\tau=0.1$ ), so the iteration will be finished. 


\section{Simulation experiments}

In this paper, we use robot Pioneer3 experiment in the fixed indoor environment. Eight sonar sensors are configured in Pioneer3, and it stops to measure data every $10 \mathrm{~cm}$. Its route can cover the whole environment, and the experiment detects multiple data samples. Figure 1 shows the robot image used in the experiment.

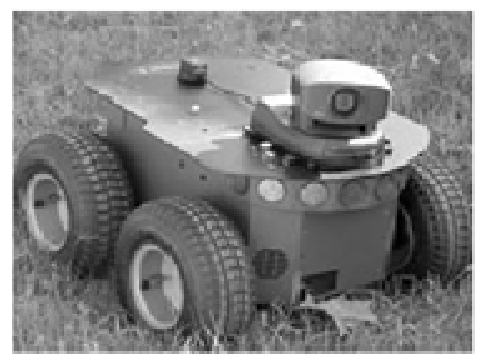

Figure 1 Pioneer3 Robot

In the original experiment, exhaustive search is commonly used to merge two local maps. When map fusion is performed, one map is fixed and the other's plane transformation is transformed. In the real environment, Pioneer 3 moves in a diversified direction, and it detects the environment from different angles. When the map is merged, the rotation angle $\theta$ must take into account a variety of situations. If you use exhaustive search method, the computation will increase greatly. In order to merge this kind of topological map, ICP registration algorithm is adopted, and the simulation experiment is conducted to compare and verify.

(1)Simulation experiment using exhaustive search

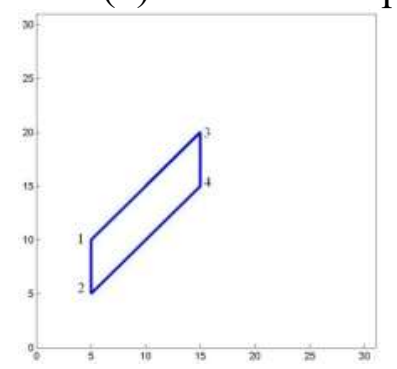

Figure 2 Original MapA of the environment Figure 3 Original MapB

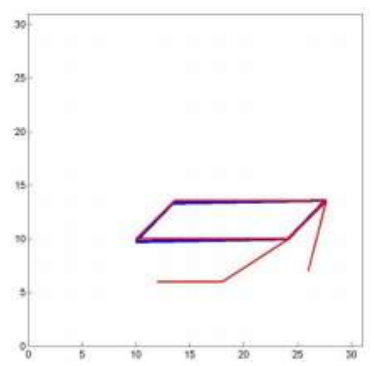

Figure 4 The merging map when $\theta=136^{\circ}$

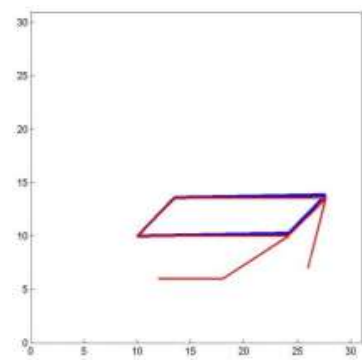

Figure $5 \theta=316^{\circ}$

Figure 2 and Figure 3 shows two original maps of the environment. Figure2 is a simpler map and Figure 3 is more complex. In the experiment, we take the initial value $\theta=0^{\circ}$ and then we change it for the step $2^{0}$. The original MapA cannot be fully integrated with MapB after plane transformation. In the condition of accepted some error, figure 4 shows that the MapA spins anticlockwise $136^{0}$ (means $\theta=136^{\circ}$ ), and that is when $\mathrm{va}_{1}$ and $\mathrm{vb}_{2}$ overlaps, the fusion of two maps is best. Figure 5 shows that the MapA spins anticlockwise $316^{\circ}$ (means $\theta=316^{\circ}$ ), and that is when $\mathrm{va}_{2}$ and $\mathrm{vb}_{1}$ overlaps, the fusion of two maps is also as the best.

During the calculation of the experiment, the procedure will be calculated once every time

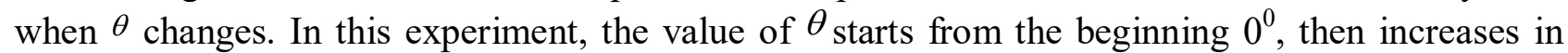
sequence $2^{0}$. After computes in sequence, 181 calculations were made by the end of the experiment. However, the MapA has 4 vertices and the MapB has 7 vertices. In total, 4 x 7 x 181=5068 fusion results are needed to determine which fusion result is the best.

(2) Simulation experiment using ICP-SVD

In order to verify the feasibility of ICP-SVD matching algorithm in topological map merging, we use MATLAB to make simulation experiment. 

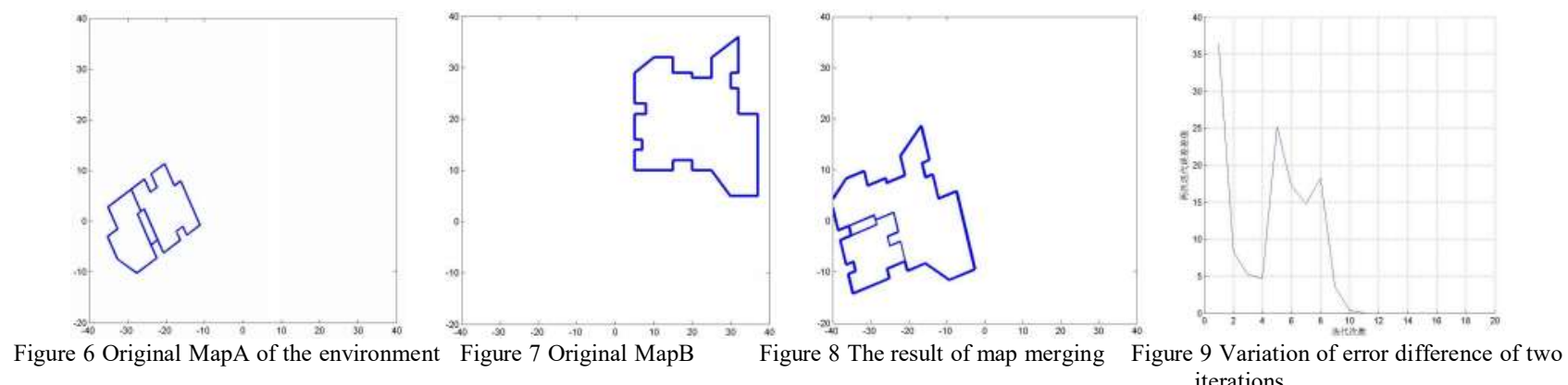

Figure 6 and Figure 7 show the original map of the environment. During the image registration, fixed the MapA as a "template", and make a plane transform of MapB. Figure 8 shows the final registration result of the two maps. From the picture we can see that, the accuracy of global map after the registration is improved greatly.

After calculation,

$$
\begin{aligned}
& \text { rotation matrix } \mathrm{R}=\left[\begin{array}{cc}
0.9540 & -0.2997 \\
0.2997 & 0.9540
\end{array}\right], \\
& \text { translation matrix } \mathrm{T}=\left[\begin{array}{l}
-36.461 \\
-25.258
\end{array}\right] .
\end{aligned}
$$

Figure 9 shows the difference of variation curve between the two successive iterations. The horizontal coordinate is the number of iterations, and the vertical coordinate is the iterative error difference of two consecutive. In this experiment, when we iterate 11 times, the iterative error of two adjacent times is less than the given threshold $(0.1)$, so the iteration ends at the same time.

The simulation results of ICP-SVD matching algorithm in topological map merging show that for the two maps in any environment, the merging map is very good. Moreover, the algorithm is simple and the time is short. This greatly improves the work efficiency of multi-mobile robot system, and the resulting environment map is more accurate.

Using the robot lab conditions of our school, the accuracy of ICP-SVD matching algorithm in topological map merging is verified in this paper (Two robots are employed in the experiment).
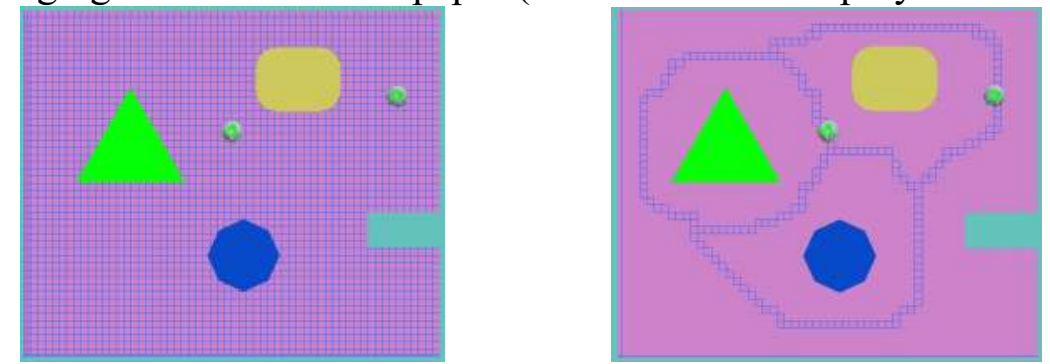

Figure 10 Laboratory environment Figure 11 Topological map of the laboratory environment

Experimental results in the robot lab show that the topological map used ICP-SVD algorithm is more effective and accurate. This reduces the time loss and meets the actual requirement.

\section{Conclusion}

In this paper, we study the topological map merging of multi-mobile robot. In the case where the robot is unknown, we use ICP-SVD algorithm to fuse two topological maps. In the MATLAB environment, the simulation of different environments is carried out, and experiments are also verified in the laboratory. The results show that ICP-SVD image registration algorithm can achieve satisfactory results. Moreover, the algorithm is stable and simple, and the time of consumption is shorter. This greatly improves the efficiency of multi-mobile robot system.

\section{Acknowledgement}

This work was supported by Science and Technology Research of Higher Education Institutions of Shandong Province. (Grant No.J14LB61) 


\section{References}

[1] Kwon T.B, Song J.B. Real-time building of a thinning-based topological map. Intelligent Service Robotics, 2008, 1(3): 211-220.

[2] Konolige K, Fox D. Map merging for distributed robot navigation. In Proceedings of the 2003 IEEE/RSJ International Conference on Intelligent Robot and Systems, 2003, vol.1: 212-217.

[3]Howard A, Sukhatme G S, Mataric M J. Multi robot simultaneous localization and mapping using manifold representations. Proceedings of the IEEE 2006: 1360-1369.

[4] Huang W.H, Beevers K.R. Topological map merging [J]. The International Journal of Robotics Research, August 2005, 24(8): 601-613. 